Supporting Information for:

\title{
Free-energy simulations support a lipophilic binding route for melatonin receptors
}

Gian Marco Elisi ${ }^{1}$, Laura Scalvini $^{1}$, Alessio Lodola ${ }^{1}$, Marco Mor $^{1,2^{*}}$, Silvia Rivara ${ }^{1}$

${ }^{1}$ Dipartimento di Scienze degli Alimenti e del Farmaco, Università degli Studi di Parma, Parco Area delle Scienze 27/A, I-43124 Parma, Italy

${ }^{2}$ Microbiome Research Hub, University of Parma, I-43124 Parma, Italy

\section{Corresponding Author}

* Dipartimento di Scienze degli Alimenti e del Farmaco, Università degli Studi di Parma, Parco Area delle Scienze 27/A, I-43124 Parma, Italy. Phone: +39 0521 905059. E-mail: marco.mor@unipr.it 


\section{S1. Docking of compound 1 in the $\mathrm{MT}_{1}$ receptor}

The crystal structure of the $\mathrm{MT}_{1}$ receptor in complex with 2-phenylmelatonin (PDB id 6ME3) $)^{1}$ was used for docking calculations after deletion of the co-crystallized ligand. The docking grid was centered on 2-phenylmelatonin, defining a bounding box of 20x10x10 $\AA$ and an enclosing box of 40x30x30 Å. Ligand docking was performed with Glide $7.9^{2,3}$ in standard precision mode and with default scaling factors, ${ }^{2}$ setting MAXKEEP and MAXREF parameters, which control the number of poses to retain after the rough scoring stage and the number of poses to refine, to 50,000 and 4000, respectively. The scoring window cutoff was increased to 1000 to widen the selection of the initial poses for the rough scoring stage. The best ligand pose according to the GScore scoring function was merged into the protein structure and the complex (Figure S1) was energy-minimized to a gradient of $0.01 \mathrm{~kJ} \cdot \mathrm{mol}^{-1} \cdot \AA^{-1}$ with OPLS3e force field ${ }^{4}$ implemented in MacroModel $12.0^{5}$ in an implicit water solvation model, ${ }^{6}$ using the Polak-Ribière conjugate gradient method. ${ }^{7}$

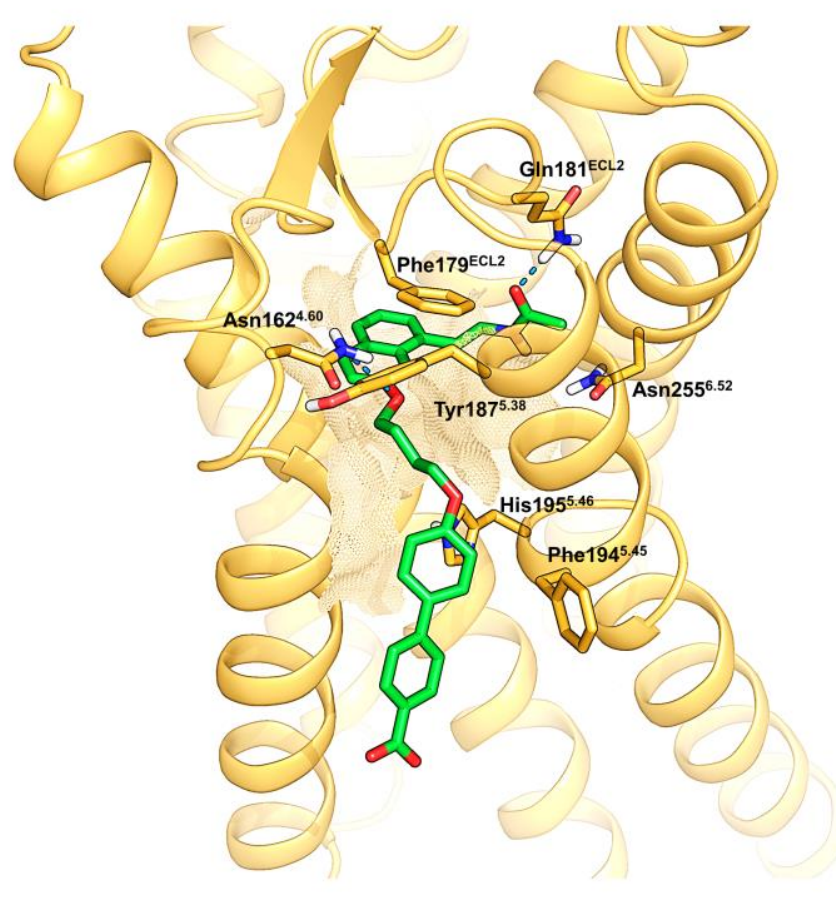

Figure S1. Docking pose of compound 1 in the $\mathrm{MT}_{1}$ receptor. The naphthalene nucleus resides in the orthosteric binding site, the amide side chain and the methoxy oxygen interact with Gln $181^{\text {ECL2 }}$ and 
Asn $162^{4.60}$, respectively, as observed for co-crystallized ligands. ${ }^{1}$ The biphenyl-carboxylate moiety is accommodated at the protein-membrane interface and protrudes through the channel between TM helices IV and V via the oxybutyloxy spacer.

\section{S2. Minimization and equilibration protocol}

The systems were minimized using the sander module implemented in Amber $16^{8}$ with three minimization cycles of 5,000 steps, each one passing from the steepest descent method to the conjugate gradient, and with protein backbone restrained with a constant of $50 \mathrm{kcal} \cdot \mathrm{mol}^{-1} \cdot \AA^{-2}$. The coordinates and the topology files of the minimized structures were then converted in the Gromacs coordinates and topology input files with the Acpype parser. ${ }^{9}$

Systems equilibration of the minimized complexes comprised different stages:

- $4.0 \mathrm{~ns}$ in NVT ensemble, ${ }^{10}$ heating from 10 to $200 \mathrm{~K}$ with a timestep of $1 \mathrm{fs}$, with restrained protein and ligand heavy atoms. The membrane was relaxed in the last $2.0 \mathrm{~ns}$, with only heteroatoms of phosphocholine restrained;

- $4.0 \mathrm{~ns}$ in NPT ensemble, ${ }^{10}$ heating from 200 to $298 \mathrm{~K}$ with a timestep of $1 \mathrm{fs}$, with restrained protein and ligand heavy atoms. During the first ns, phosphocholine heteroatoms were also restrained;

- $12.0 \mathrm{~ns}$ in NPT ensemble, with protein and ligand heavy atoms progressively relaxed, until only the alpha carbons were restrained with a spring constant of $0.1 \mathrm{kcal} \cdot \mathrm{mol}^{-1} \cdot \AA^{-2} \cdot 11,12,13,14$

These steps were followed by two $\mathrm{NVT}^{13,14}$ stages restraining the positions of alpha carbons to those of the crystal structures:

- $\quad 100.0 \mathrm{~ns}$ in NVT ensemble, with alpha carbons restrained with a spring constant of 0.1 $\mathrm{kcal} \cdot \mathrm{mol}^{-1} \cdot \AA^{-2}$. The output structure of this NVT stage was used as input structure for bidimensional metadynamics (Paragraph S7). 
- $\quad 100.0$ ns in NVT ensemble, with only 89 selected backbone carbons (see Paragraph S3 for backbone atoms selection) restrained with a spring constant of $0.1 \mathrm{kcal} \cdot \mathrm{mol}^{-1} \cdot \AA^{-2}$. The output structure of this NVT stage was used as input structure for all the remaining enhanced sampling simulations and for $\mathrm{MD}$ simulation of the $\mathrm{MT}_{1}$ receptor in complex with compound 1 .

\section{S3. Definition of restrained backbone carbons}

89 carbons were restrained in $\mathrm{MT}_{1}$ and $\mathrm{MT}_{2}$ receptors, comprising 5 carbons on the truncated $\mathrm{N}-$ terminal tip (the carbonyl carbon of the acyl-cap and alpha carbons of the first four residues) and 84 alpha carbons belonging to three consecutive turns in the intracytoplasmic side of each TM helix (12 alpha carbons for each helix). The following alpha carbons were restrained:

\begin{tabular}{llll}
\multicolumn{2}{c}{ MT $_{1}$ receptor } & \multicolumn{2}{l}{ MT $_{2}$ receptor } \\
N-term: & Pro23-Leu26 & N-term: & Pro36-Val39 \\
TM I: & Leu43-Arg54 & TM I: & Val56-Arg67 \\
TM II: & Ile64-Val75 & TM II: & Leu77-Val88 \\
TM III: & Ile123-Lys134 & TM III: & Ile136-Ala147 \\
TM IV: & Ser144-Thr155 & TM IV: & Thr157-Thr168 \\
TM V: & Tyr207-Arg218 & TM V: & Tyr220-Arg231 \\
TM VI: & Arg235-Leu246 & TM VI: & Arg248-Ile259 \\
TM VII: & Asn287-Leu298 & TM VII: & Asn300-Leu311
\end{tabular}

\section{S4. Unbinding CV for steered molecular dynamics: definition of plane $\eta$}

Plane $\eta$ is defined through the coordinates of three centers of mass, $\mathrm{H}_{1}, \mathrm{H}_{2}$ and $\mathrm{H}_{3}$, defined in counterclockwise order, observing from the outside of the receptor, as in Figure 2 of the main text. The centers of mass are related to the following alpha carbons:

$\underline{\mathrm{MT}}_{1}$ receptor

H1: Ser144-Thr155

H2: Tyr207-Arg218

H3: $\overbrace{\text { Leu156 - Leu163 }}^{T M I V}, \overbrace{\text { Thr188 - His195 }}^{T M V}$
$\underline{\mathrm{MT}}_{2}$ receptor

H1: Thr157-Thr168

H2: Tyr220-Arg231

H3: $\overbrace{\text { Val169 - Phe176 }}^{T M I V}, \overbrace{\text { Thr201- His208 }}^{T M V}$ 


\section{S5. Path CVs (PCVs) optimization: technical details}

Path optimization ${ }^{15}$ was performed through four consecutive steered molecular dynamics (SMD) simulations ${ }^{16}$ of $30.0 \mathrm{~ns}$ in which a harmonic restraint of $50 \mathrm{kcal} \cdot \mathrm{mol}^{-1}$ was applied on the $\mathcal{S} \mathrm{CV}$, restricting the access to configurations of the system distant from the reference frameset by restraining the exploration of the $\mathcal{Z} \mathrm{CV}$ with a force constant of 1,000 $\mathrm{kcal} \cdot \mathrm{mol}^{-1} \cdot \AA^{-4}$ at $Z=1 \AA^{2}$. In these simulations, $\lambda$ was tuned to assure a smooth transition between coordinates of the system corresponding to reference configurations with the highest inter-frame distance in the region of the $\mathrm{CV}(\mathcal{S}=1-38)$, in which the ligand interacts with the receptor $\left(\lambda_{1}=1.05 \AA^{-2}, \lambda_{2}=1.87 \AA^{-2}, \lambda_{3}=2.00 \AA^{-2}, \lambda_{4}=2.08 \AA^{-2}\right)$.

Between each pair of the four SMD-PCV runs, the reference configurations were iteratively updated using in-house scripts. A procedure using the Catmull-Rom splines method ${ }^{17}$ was set up to generate additional configurations to obtain a smoother guess path, by interpolating the SMD trajectory through the insertion of ten intermediate configurations between each pair of consecutive SMD frames. The 50 reference configurations were then extracted, on the basis of an RMSD-distance matrix calculated on the whole frameset, through an operation based on the Nudged Elastic Band (NEB) approach; ${ }^{18}$ in this procedure, the Monte Carlo sampling was used to search for the best sequence of configurations describing transition states regions. 10 iterations of the NEB algorithm were performed using a Metropolis coefficient of 10,000, keeping the endpoints (first and last reference configurations) fixed. After every SMD-PCV simulation, the set of frames thus obtained was employed as the new (and refined) reference path for a subsequent SMD-PCV run. In three consecutive runs, the work profile stabilized around similar values (Figure S2). The reference configurations within $\mathcal{S}$ ranges $2-5$ and 38-50 were further optimized through targeted dynamics simulations setting as a collective variable the difference of the RMSD between the first and last configuration of each range. ${ }^{19}$ 


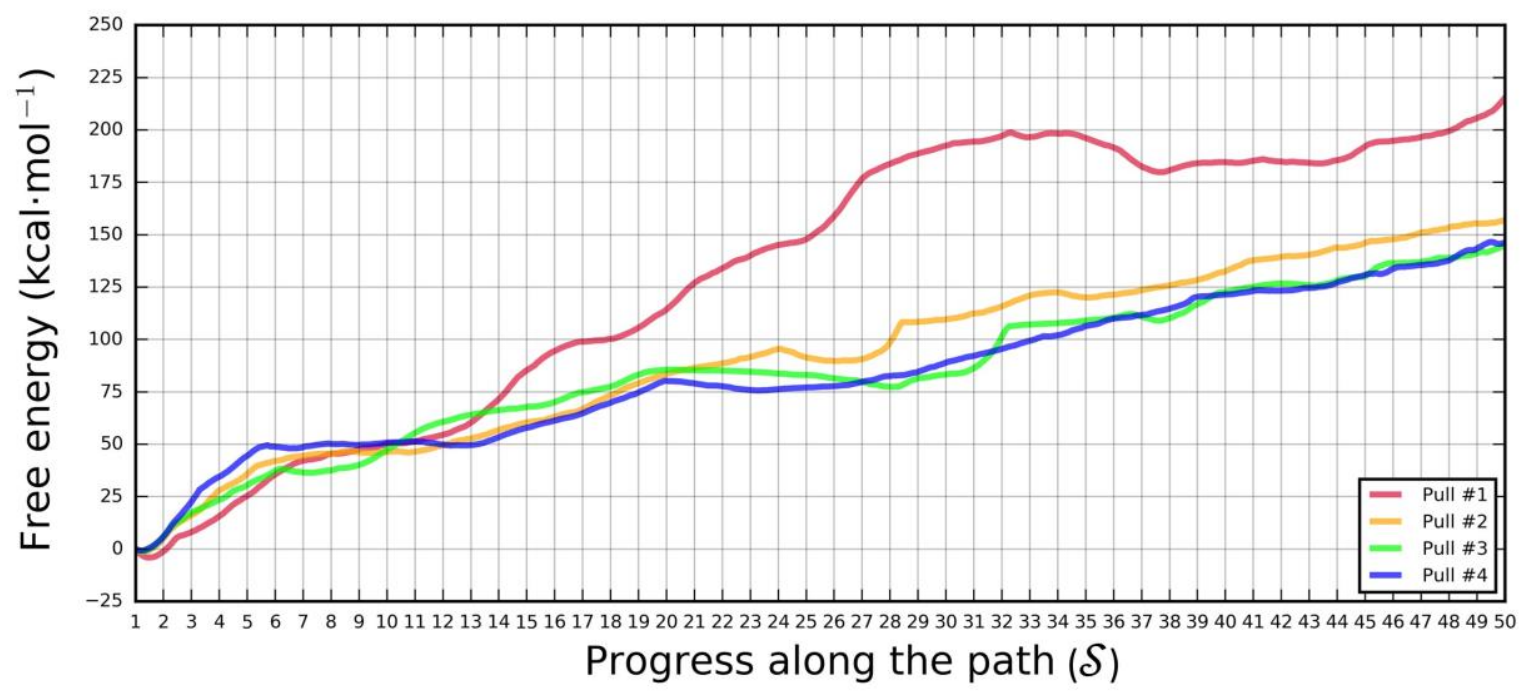

Figure S2. Work profiles of the four consecutive SMD-PCV simulations performed to optimize the path reference configurations. 


\section{S6. Analysis of PCV-US simulations}

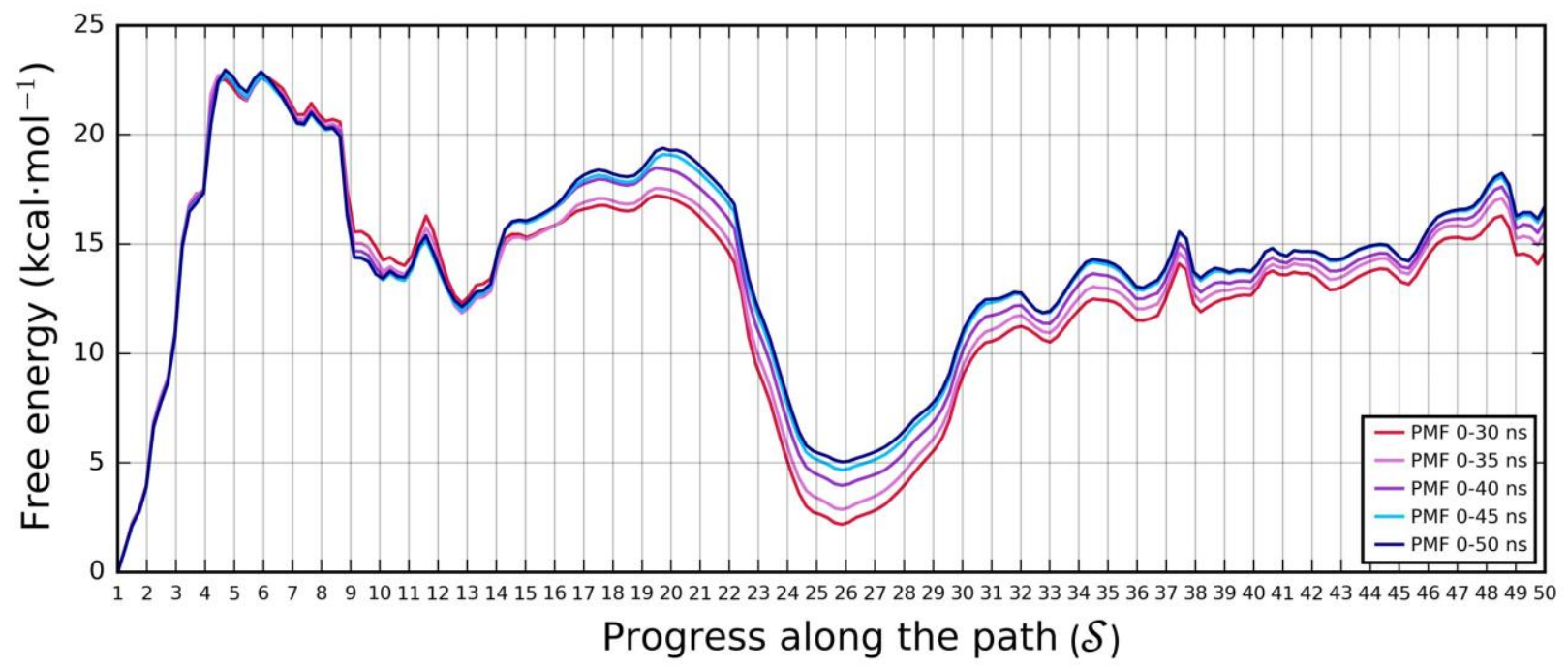

Figure S3. Free-energy profile obtained for PCV-US simulations as a function of the simulation time of each US window. Potential of mean force (PMF) curves were calculated using the WHAM algorithm, ${ }^{20,21}$ by renormalizing the probabilities for each window into a single composite probability.

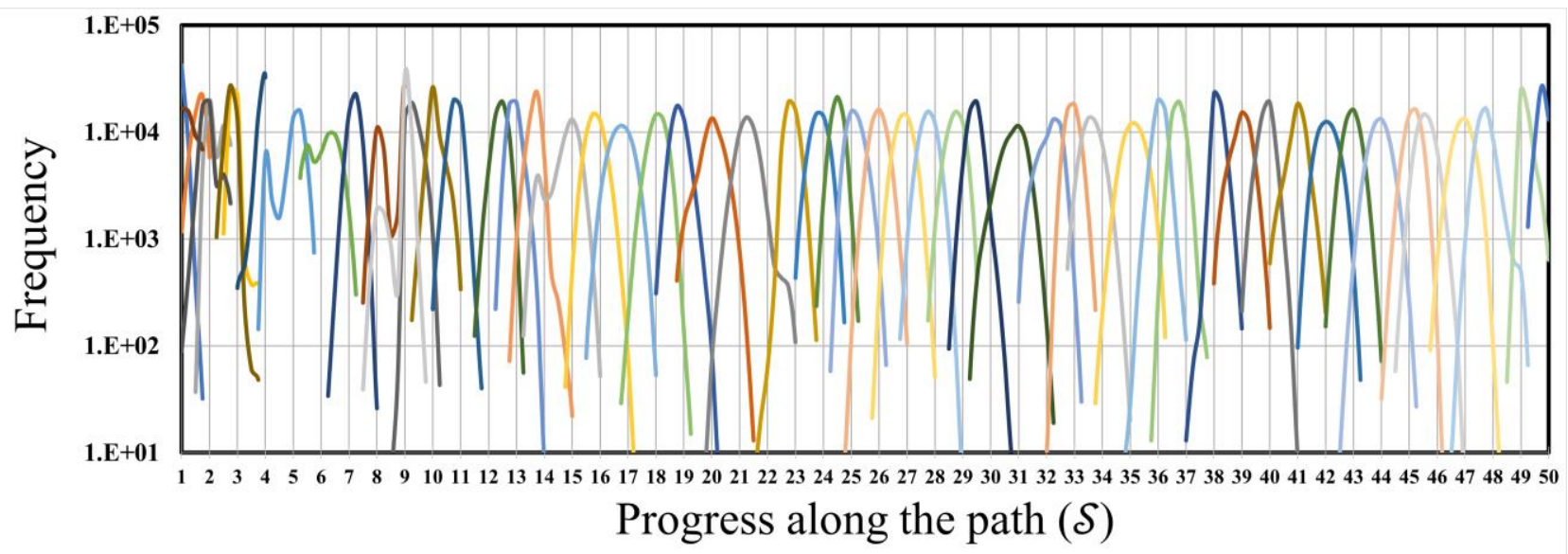

Figure S4. Distribution (log scale) of $\mathcal{S}$ values of PCV-US simulations. The value of the $\mathcal{S}$ coordinate was registered every ps for each simulation. 


\section{Qualitative agreement of the US windows a priori probabilities}

The global PMF, given by the contributions of all the US windows employed in the WHAM calculation, is a free-energy profile over the coordinate $\mathcal{S}$, divided in intervals $\mathrm{j}$ :

$$
P M F_{j}=-k_{B} T \cdot \ln p_{0_{j}}+\mathrm{F}
$$

where the constant $\mathrm{F}$ is fixed to have $\operatorname{PMF}(\mathcal{S}=1)=0 \mathrm{kcal} \cdot \mathrm{mol}^{-1}$.

The reliability of PMF was evaluated through the qualitative agreement between a priori probabilities of neighboring US windows (Figure S5). The a priori probability $p_{0_{j}}$ is related to the biased probability $p_{i j}$ that the $\mathrm{i}^{\text {th }}$ simulation falls into the $\mathrm{j}^{\text {th }}$ interval according to the following relationship:

$$
P_{i j}=P_{0_{j}} \cdot c_{i j} \cdot f_{i}
$$

where $c_{i j}$ represents the probability distortion due to the bias potential and $f_{i}$ is a normalization coefficient.

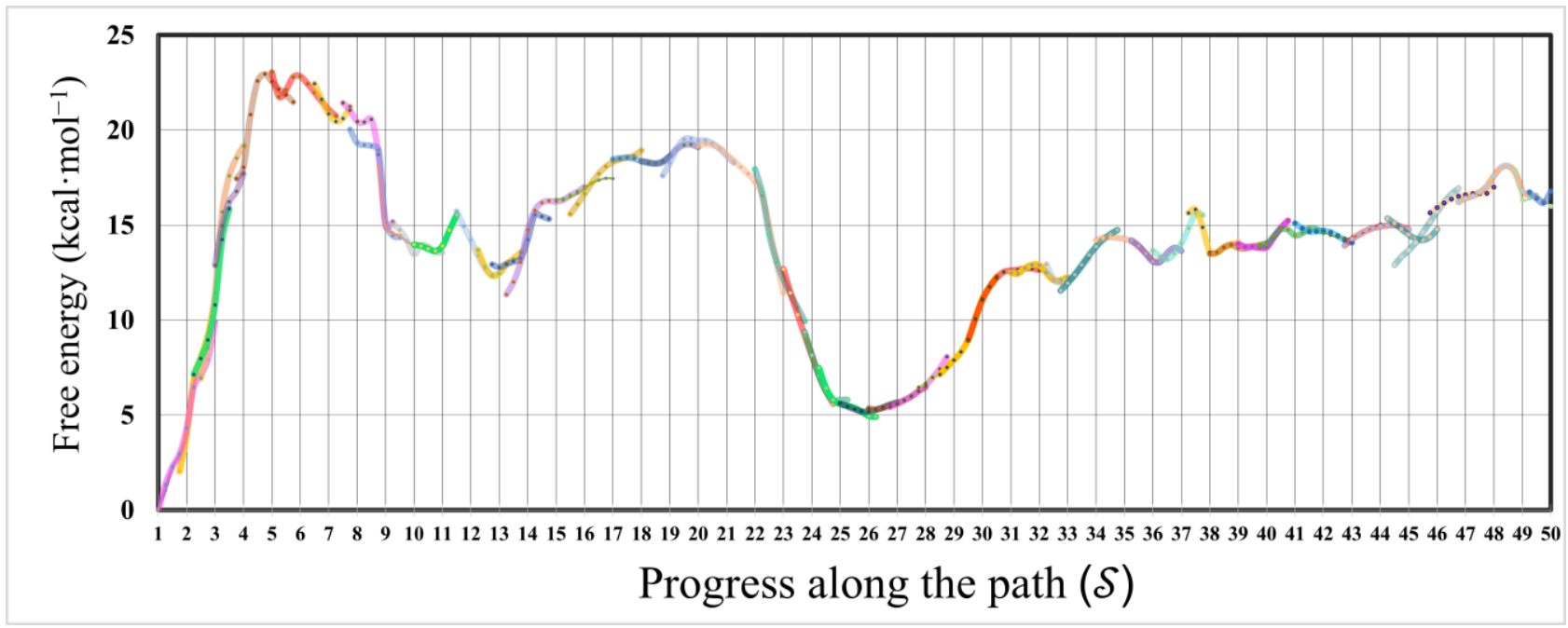

Figure S5. Dissection of the global potential of mean force (PMF) into the free-energy profiles of the PCV-US windows, scaled as free-energy differences with respect to $\mathcal{S}=1$ (bound state). 


\section{S7. Well-tempered metadynamics simulations on Tyr187/200 5.38}

Table S1. Parameters adopted for monodimensional and bidimensional well-tempered metadynamics simulations on Tyr187/200 5.38 dihedral angles.

\begin{tabular}{|c|c|c|}
\hline Parameters & 1D & 2D \\
\hline $\boldsymbol{\sigma}\left(\chi_{\mathbf{1}}\right)$ & $3^{\circ}$ & $5^{\circ}$ \\
\hline $\boldsymbol{\sigma}\left(\chi_{\mathbf{2}}\right)$ & -- & $10^{\circ}$ \\
\hline $\boldsymbol{h}_{\mathbf{0}}$ & $0.2 \mathrm{kcal} \cdot \mathrm{mol}^{-1}$ & $0.2 \mathrm{kcal} \cdot \mathrm{mol}^{-1}$ \\
\hline $\boldsymbol{\tau}$ & $3 \mathrm{ps}$ & $5 \mathrm{ps}$ \\
\hline $\boldsymbol{\gamma}$ & 15 & 30 \\
\hline restraints & $\begin{array}{c}89 \text { atoms } \\
\text { (see Paragraph S3) }\end{array}$ & all alpha carbons \\
\hline
\end{tabular}

\section{Bidimensional well-tempered metadynamics simulations}

Bidimensional well-tempered metadynamics simulations ${ }^{22}$ were performed with restrained receptor alpha carbons. $\chi_{2}$ dihedral angle was compactified in a $[0, \pi]$ interval with a Heaviside function to avoid the exploration of symmetric degrees of freedom. FES are shown after 1.0 and $1.1 \mu$ s of simulation (Figure S6). For each system, the exploration of different minima across the CVs and the time-evolution of potentials deposition was assessed (Figure S7). 


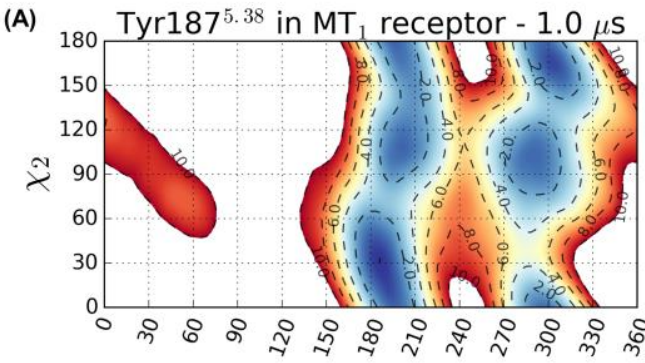

$\chi_{1}$

(C)

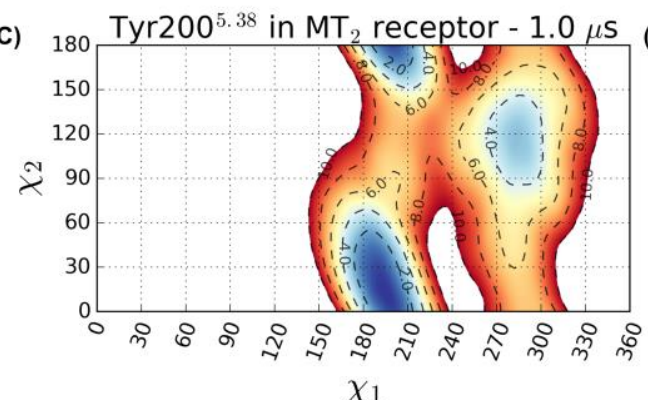

(B)

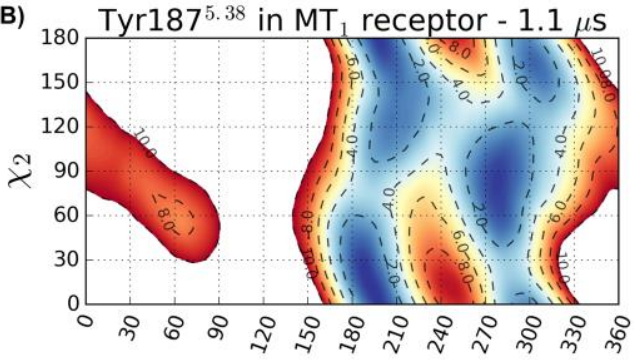

$\chi_{1}$

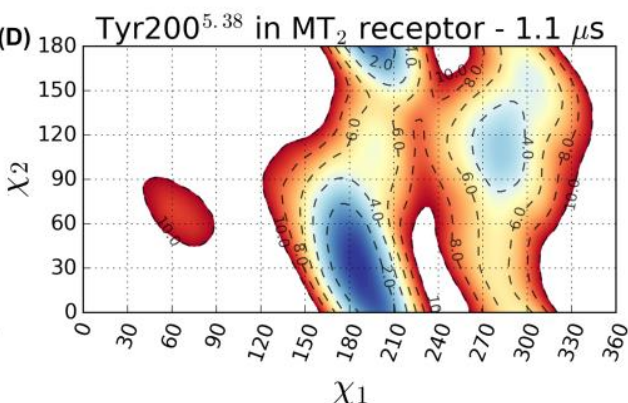

Figure S6. Free-energy surfaces for Tyr5.38 side chain after 1.0 (A and $\mathbf{C})$ and $1.1 \mu$ s $(\mathbf{B}$ and D) of well-tempered metadynamics simulations performed on dihedral angles $\chi_{1}$ and $\chi_{2}$ of the $\mathrm{MT}_{1}(\mathbf{A}$ and $\mathbf{B})$ and $\mathrm{MT}_{2}(\mathbf{C}$ and $\mathbf{D})$ receptors in complex with 2-iodomelatonin.

(A)



(C)

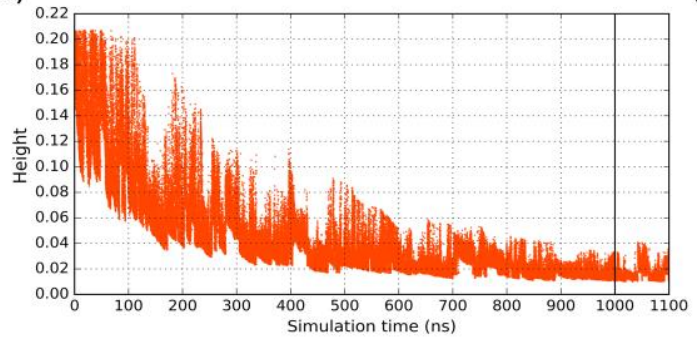

(B)

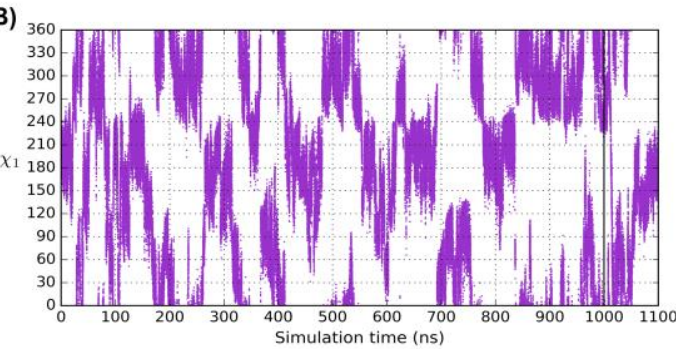

(D)

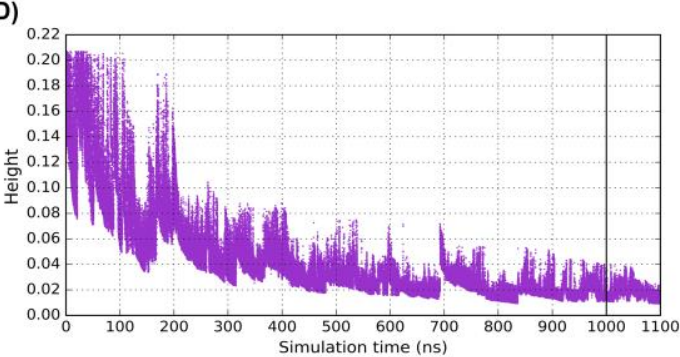

Figure S7. Time-evolution of the values assumed by the $\chi_{1}$ dihedral angle in $\mathrm{MT}_{1}(\mathbf{A})$ and $\mathrm{MT}_{2}$

(B) receptors in complex with 2 -iodomelatonin. $\chi_{2}$ dihedral angle is not shown as it is a 
completely diffusive CV. Time-evolution of the height of the Gaussian potentials deposited in the two systems ( $\mathbf{C}$ and $\mathbf{D})$.

\section{S8. Analysis of Tyr187/200 5.38 side chain conformation during ligand unbinding}

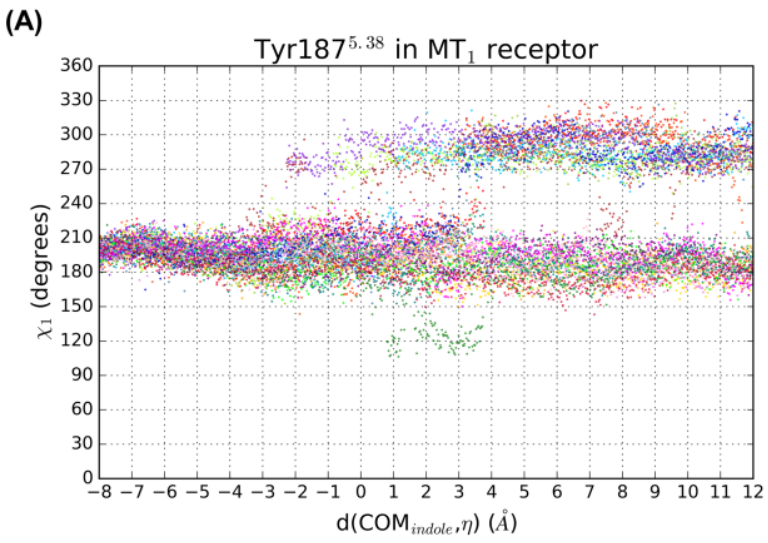

(B)

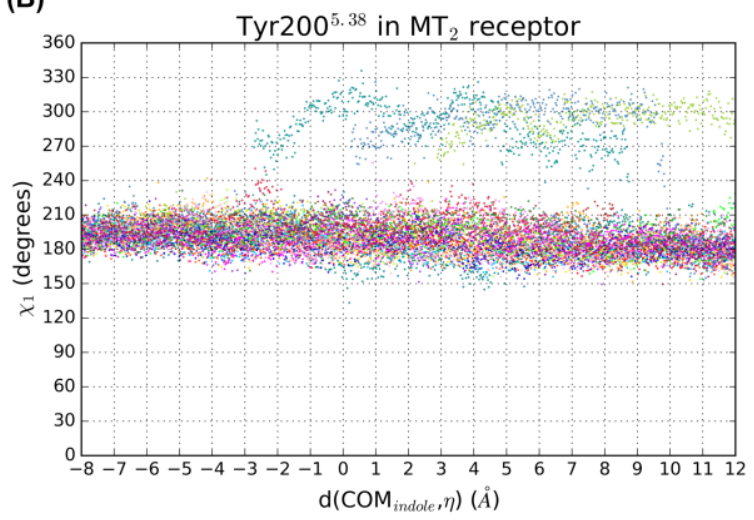

Figure S8. Conformation of Tyr187/200 ${ }^{5.38}$ in SMD simulations. Values of Tyr5.38 $\chi_{1}$

dihedral angle monitored in the twenty SMD simulations during the unbinding of 2iodomelatonin from the $\mathrm{MT}_{1}(\mathbf{A})$ and the $\mathrm{MT}_{2}(\mathbf{B})$ receptors. Each color represents a different simulation. The open state of Tyr5.38 side chain $\left(270^{\circ}<\chi_{1}<300^{\circ}\right)$ is more populated in simulations of ligand exiting from the $\mathrm{MT}_{1}$ receptor than from the $\mathrm{MT}_{2}$ receptor. $\chi_{1}$ dihedral angle assumed values around $300^{\circ}$ in ten out of twenty simulations of the $\mathrm{MT}_{1}$-receptor complex, and in 3 simulations of the $\mathrm{MT}_{2}$-receptor complex. 


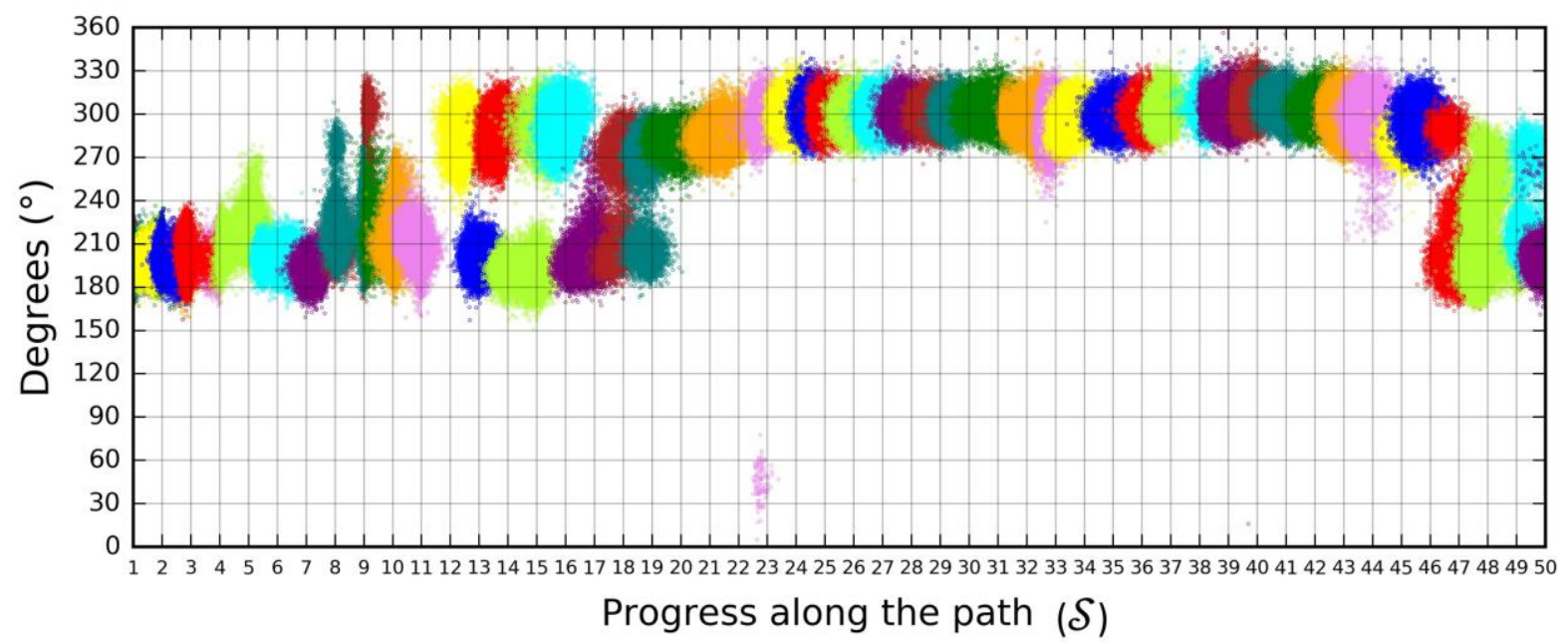

Figure S9. Conformation of $\mathrm{MT}_{\mathbf{1}}$ Tyr187 ${ }^{5.38}$ in PCV-US simulations. Values of Tyr $187^{5.38}$ $\chi_{1}$ dihedral angle are monitored every 2 ps in PCV-US simulations of 2-iodomelatonin unbinding from the $\mathrm{MT}_{1}$ receptor. Each color represents an independent simulation of $50 \mathrm{~ns}$.

\section{References}

1 Stauch, B.; Johansson, L. C.; McCorvy, J. D.; Patel, N.; Han, G. W.; Huang, X.; Gati, C.; Batyuk, A.; Slocum, S. T.; Ishchenko, A.; Brehm, W.; White, T. A.; Michaelian, N.; Madsen, C.; Zhu, L.; Grant, T. D.; Grandner, J. M.; Shiriaeva, A.; Olsen, R. H. J.; Tribo, A. R.; Yous, S.; Stevens, R. C.; Weierstall, U.; Katritch, V.; Roth, B. L.; Liu, W.; Cherezov, V. Structural basis of ligand recognition at the human $\mathrm{MT}_{1}$ melatonin receptor. Nature 2019, 569, 284-288. 
2 Friesner, R. A.; Banks, J. L.; Murphy, R. B. Glide: A new approach for rapid, accurate docking and scoring. 1. Method and assessment of docking accuracy. J. Med. Chem. 2004, 47, 1739-1749.

3 Schrödinger Release 2018-2: Glide 7.9, Schrödinger, LLC, New York, NY, 2018.

4 Roos, K.; Wu, C.; Damm, W.; Reboul, M.; Stevenson, J. M.; Lu, C.; Dahlgren, M. K.; Mondal, S.; Chen, W.; Wang, L.; Abel, R.; Friesner, R. A.; Harder, E. D. OPLS3e: Extending force field coverage for drug-like small molecules. J. Chem. Theory Comput. 2019, 15, 18631874.

5 Schrödinger Release 2018-2: MacroModel 12.0, Schrödinger, LLC, New York, NY, 2018.

6 Still, W. C.; Tempczyk, A.; Hawley, R. C. Semianalytical treatment of solvation for molecular mechanics and dynamics. J. Am. Chem. Soc. 1990, 112, 6127-6129.

7 Polak, E.; Ribière, G. Note sur la convergence de méthodes de directions conjuguées. Revue Française Inf. Rech. Opér. Sér. rouge 1969, 16, 35-43.

8 Case, D. A.; Betz, R. M.; Cerutti, D. S.; Cheatham, III, T. E.; Darden, T. A.; Duke, R. E.; Giese, T. J.; Gohlke, H.; Goetz, A. W.; Homeyer, N.; Izadi, S.; Janowski, P.; Kaus, J.; Kovalenko, A.; Lee, T. S.; LeGrand, S.; Li, P.; Lin, C.; Luchko, T.; Luo, R.; Madej, B.; Mermelstein, D.; Merz, K. M.; Monard, G.; Nguyen, H.; Nguyen, H. T.; Omelyan, I.; Onufriev, A.; Roe, D. R.; Roitberg, A.; Sagui, C.; Simmerling, C. L.; Botello-Smith, W. M.; Swails, J.; Walker, R. C.; Wang, J.; Wolf, R. M.; Wu, X.; Xiao, L.; Kollman P. A. AMBER 2016, University of California, San Francisco 2016. 
9 Sousa da Silva, A. W.; Vranken, W. F. ACPYPE - AnteChamber PYthon Parser interfacE. BMC Res. Notes 2012, 5, 367.

10 Berendsen, H. J. C.; Postma, J. P. M.; van Gunsteren W. F.; Di Nola, A.; Haak, J. R. Molecular dynamics with coupling to an external bath. J. Chem. Phys. 1984, 81, 3684-3690.

11 Nosé S. A Molecular Dynamics Method for Simulations in the Canonical Ensemble. Mol. Phys. 1984, 52, 255-268.

12 Hoover WG. Canonical dynamics: Equilibrium phase-space distributions. Phys. Rev. A 1985, 31, 1695.

13 Parrinello, M.; Rahman, A. Polymorphic transitions in single crystals: a new molecular dynamics method. J. Appl. Phys. 1981, 52, 7182-7190.

14 Nose, S.; Klein, M. L. Constant pressure molecular dynamics` for molecular systems. Mol. Phys. 1983, 50, 1055-1076.

15 Branduardi, D.; Gervasio, F. L.; Parrinello, M. From A to B in free energy space. $J$. Chem. Phys. 2007, 126, 054103.

16 Grubmüller, H.; Heymann, B.; Tavan, P. Ligand Binding: Molecular Mechanics Calculation of the Streptavidin-Biotin Rupture Force. Science 1996, 271, 997-999.

17 Catmull, E.; Rom, R. A class of local interpolating splines. In Computer aided geometric design, 1st ed..; New York: Academic Press. In: Barnhill R. E. \& Riesenfeld R. F., 1974; pp. 317-332. 
18 Jónsson, H.; Mills, G.; Jacobsen, K. W. Classical Dynamics in Condensed Phase Simulations. In: B. J. Berne, G. Ciccotti, and D. F. Coker; World Scientific, Singapore, 1998; p. $385-404$.

19 Favia, A. D.; Masetti, M.; Recanatini, M.; Cavalli, A. Substrate binding process and mechanistic functioning of type $111 \beta$-hydroxysteroid dehydrogenase from enhanced sampling methods. PLoS One 2011, 6, e25375.

20 Kumar, S.; Rosenberg, J. M.; Bouzida, D.; Swendsen, R. H., Kollman, P. A. The weighted histogram analysis method for free-energy calculations on biomolecules. I. The method. J. Comput. Chem. 1992, 13, 1011-1021.

21 Grossfield A. "WHAM: the weighted histogram analysis method", version 2.0.9, http://membrane.urmc.rochester.edu/wordpress/?page_id=126.

22 Barducci, A.; Bussi, G.; Parrinello, M. Well-Tempered Metadynamics: a smoothly converging and tunable free-energy method. Phys. Rev. Lett. 2008, 100, 020603. 